\title{
DISCUSSION
}

\section{Slope stability analysis by finite elements}

\author{
D. V. GRIFFITHS \& P. A. LANE (1999). Géotechnique 49, No. 3, 387-403
}

F. Cai and K. Ugai. Gunma University, Japan

The authors have presented various numerical comparisons of the safety factor of slopes between the finite element method (FEM) and limit equilibrium method (LEM) under two-dimensional conditions. They conclude that the FEM with an elastoplastic (Mohr-Coulomb) constitutive model is a reliable and robust approach to evaluating the safety factor of slopes. The main advantage of the FEM is that the safety factor emerges naturally from the analysis without the user having to commit to any particular form of the mechanism a priori.

We have been interested in the FEM to predict the safety factor of slopes for a long time (Ugai, 1989). The FEM procedure is very similar to that reported by the authors; here it is called the shear strength reduction FEM. This procedure can predict the safety factor of slopes within a single framework for both two- and three-dimensional problems. The FEM can easily be used to investigate three-dimensional situations, such as the excavated pit (Ugai \& Leshchinsky, 1995), slopes with horizontal drains to lower the groundwater level under rainfall (Cai et al., 1998), or slopes reinforced with piles (Cai \& Ugai, 1999). In general, it has been difficult for the LEM to locate the critical slip surface under three-dimensional conditions. The reliability of the LEM may be in doubt in the case of soilstructure interaction. This will be made clear with the following example.

When the three-dimensional shear strength reduction FEM is used to analyse the stability of an idealised slope reinforced with piles, the soil-pile interaction is simulated by zero-thickness elasto-plastic (Mohr-Coulomb) interface elements. The FE mesh is shown in Fig. 22. Two symmetrical boundaries are used, so that the problem analysed actually consists of a row of piles with planes of symmetry through the pile centreline and through the soil midway between piles. The material properties of the soil, pile and interface are listed in Table 3. The influence of the pile position, $L_{x}$, on the safety factor of slopes, $F_{\mathrm{s}}$, is shown in Fig. 23. The results determined by the FEM are compared with those found by the LEM (Bishop's simplified method). In the LEM, the reaction forces of the piles are

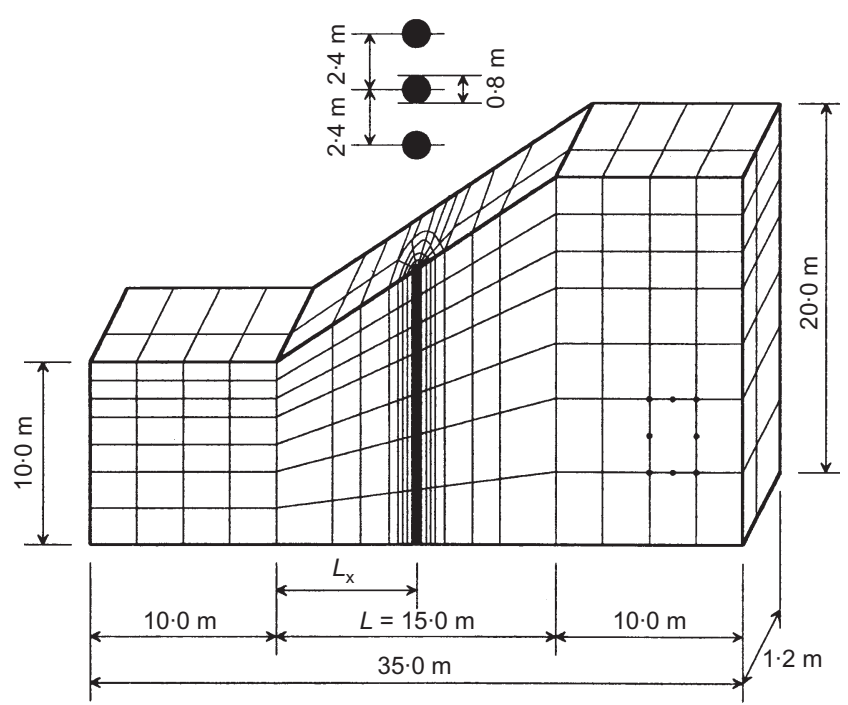

Fig. 22. An idealised slope and its FE mesh
Table 3. Material properties

\begin{tabular}{l|c|c|c}
\hline \multicolumn{1}{c|}{ Parameter } & Soil & Interface & Pile \\
\hline$c: \mathrm{kPa}$ & $10 \cdot 0$ & $10 \cdot 0$ & \\
$\phi:$ degrees & $20 \cdot 0$ & $20 \cdot 0$ & \\
$\psi:$ degrees & $0 \cdot 0$ & $0 \cdot 0$ & \\
$E: \mathrm{MPa}$ & 200 & 200 & 60000 \\
$\nu$ & $0 \cdot 25$ & $0 \cdot 25$ & $0 \cdot 20$ \\
$\gamma: \mathrm{kN} / \mathrm{m}^{3}$ & $20 \cdot 0$ & & \\
\hline
\end{tabular}

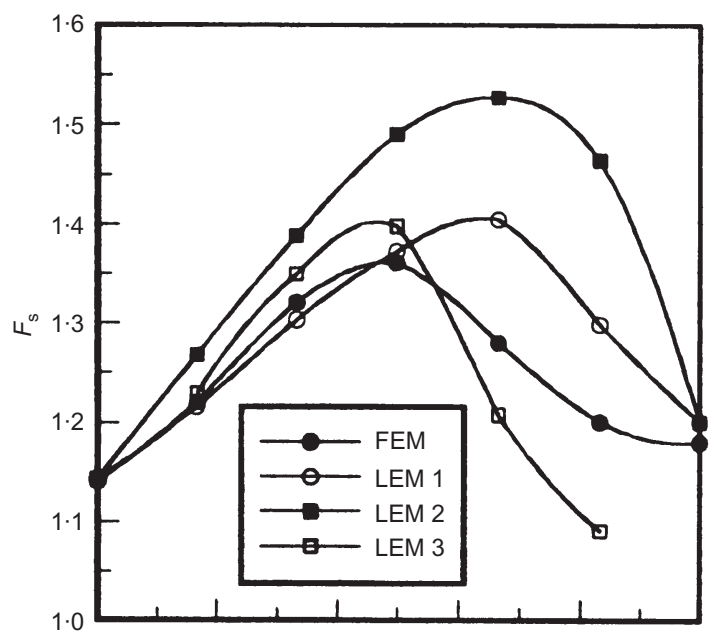

(a)

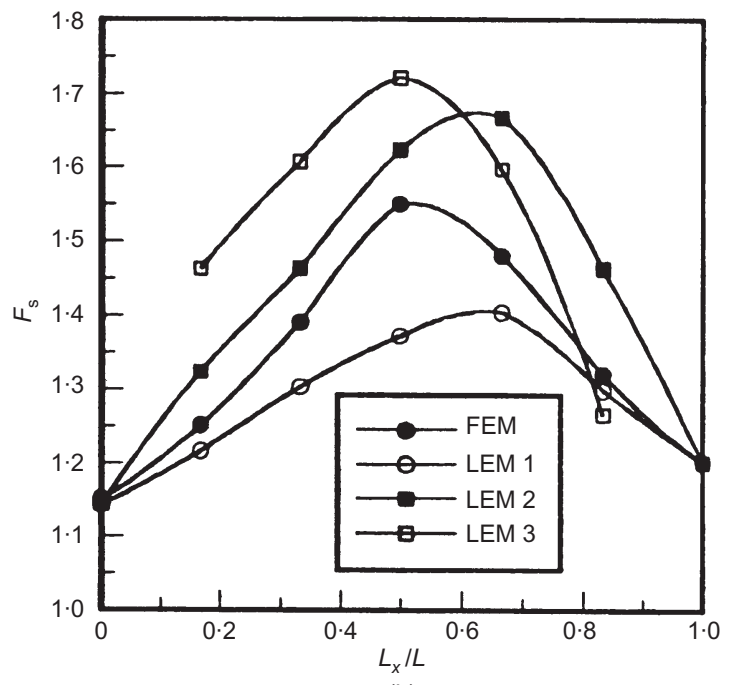

(b)

Fig. 23. Safety factor versus pile positions: (a) free-head pile; (b) hinged-head pile

determined by two methods: (1) by the equation proposed by Ito \& Matsui (1975) for LEM1 and LEM2, and (2) by the FEM results for LEM3. The minimum global safety factors are obtained by two assumptions for the slip circles: (1) in LEM1, no limits are placed on the location of the slip circles, and (2) in LEM2 and LEM3, the slip circles must pass through a 
critical depth at the pile position. The critical depth is defined as the first extreme point of shear force in the pile from its top, obtained by the FEM results. This definition is valid because the analytical solution of the piles under moving soil shows that the slip surface passes through the critical depth (Poulos, 1995; Hassiotis et al., 1997). Fig. 23 indicates that there is a significant difference between the safety factors predicted by the FEM and LEM, even when the reaction forces, obtained by the FEM results, are used in the LEM. Fig. 24 shows the displacement vectors from the FEM and the slip surfaces of LEM1, LEM2, and LEM3 with a solid line, broken line, and dotted broken line respectively. The displacement vectors indicate that the slip surface may not be continuous when the slope is reinforced with piles. This situation cannot be sufficiently considered by the LEM.

The FEM is very useful for evaluating the safety factor of slopes reinforced with piles. We think that the safety factor of slopes, predicted by the FEM, is more reliable than that obtained by the LEM, because the soil-pile interaction is taken into consideration in the FEM.

\section{Authors' reply}

The authors wish to thank the discussers for their comments and interest in our paper.

It is true that the potential of the finite element method for studying slope stability was recognised some years ago. For example, Smith \& Hobbs (1974) were among the first to apply elasto-plastic constitutive models and finite elements to stability problems. Their paper also includes a useful review of other publications on that topic.

Complex boundary value problems involving stability, especially in 3-D, often defy meaningful analysis by simplified approaches such as limit equilibrium. The discussers have effectively presented such an example involving a pile-reinforced earth slope. Their solutions provide both estimates of the factor of safety and physical insight into the mechanisms and deformations.

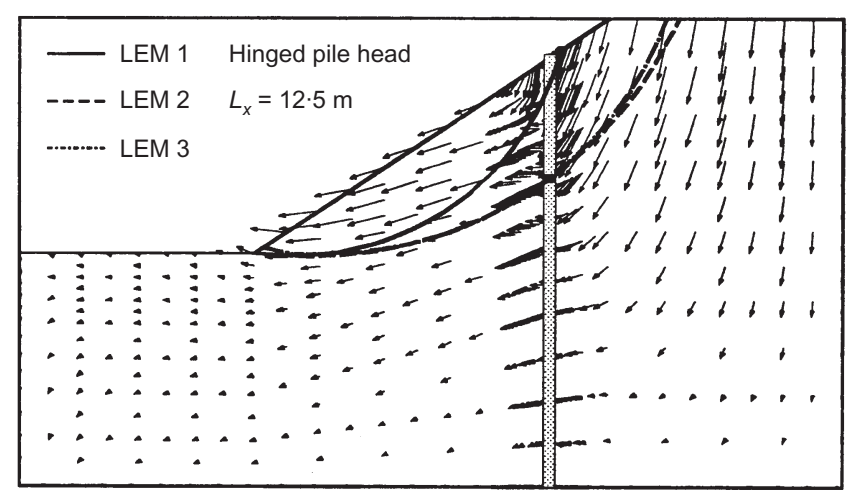

Fig. 24. Displacement vectors just before divergence by FEM and the slip surfaces by LEM

\section{REFERENCES}

Cai, F. \& Ugai, K (1999). Numerical analysis of the stability of a slope reinforced with piles. Soils Found. (submitted).

Cai, F., Ugai, K., Wakai, A. \& Li, Q. (1998). Effects of horizontal drains on slope stability under rainfall by three-dimensional finite element analysis. Comput. Geotech. 23, 255-275.

Hassiotis, S., Chameau, J. L. \& Gunaratne, M. (1997). Design method for stabilization of slopes with piles. J. Geotech. Geoenvir. Engng, ASCE 123, No. 4, 314-323.

Ito, T. \& Matsui, T. (1975). Methods to estimate lateral force acting on stabilizing piles. Soils Found. 15, No. 4, 43-59.

Poulos, H. G. (1995). Design of reinforcing piles to increase slope stability. Can. Geotech. J. 23, 808-818.

Smith, I. M. \& Hobbs, R. (1974). Finite element analysis of centrifuged and built-up slopes. Géotechnique, 24, No. 4, 531-559.

Ugai, K. (1989). A method of calculation of global safety factor of slopes by elasto-plastic FEM. Soils Found. 29, No. 2, 190-195 (in Japanese).

Ugai, K. \& Leshchinsky, D. (1995). Three-dimensional limit equilibrium and finite element analyses: a comparison of results. Soils Found. 35, No. 4, 1-7. 